\title{
Socioeconomic inequalities in risk of congenital anomaly
}

\author{
M Vrijheid, H Dolk, D Stone, L Abramsky, E Alberman, J E S Scott
}

Environmental Epidemiology Unit, Department of Public Health and Policy, London School of Hygiene and Tropical Medicine, Keppel Street, London WC1E 7HT, UK $M$ Vrijheid H Dolk

Glasgow Eurocat Register, Paediatric Epidemiology and Community Health Unit, Department of Child Health,

University of Glasgow,

Scotland, UK

D Stone

North Thames (West) Congenital

Malformation

Register, Department of Medical and Community Genetics, Imperial School of Medicine, Northwick Park Hospital, Harrow, UK

L Abramsky

National Downs Syndrome Cytogenetic Register, The Wolfson Institute of Preventive Medicine,

St Bartholomew's and the Royal School of Medicine and Dentistry, London, UK E Alberman

Northern Congenital Abnormality Survey, Maternity Survey Office, University of Newcastle-upon-Tyne, UK

J E S Scott

Correspondence to: Ms Vrijheid

email:

mvrijheid@1shtm.ac.uk

Accepted 4 February 2000

\section{Abstract}

Aims-To investigate socioeconomic inequalities in the risk of congenital anomalies, focusing on risk of specific anomaly subgroups.

Methods-A total of 858 cases of congenital anomaly and 1764 non-malformed control births were collected between 1986 and 1993 from four UK congenital malformation registers, for the purposes of a European multicentre case control study on congenital anomaly risk near hazardous waste landfill sites. As a measure of socioeconomic status, cases and controls were given a value for the area level Carstairs deprivation index, by linking the postcode of residence at birth to census enumeration districts (areas of approximately 150 households).

Results-Risk of non-chromosomal anomalies increased with increasing socioeconomic deprivation. The risk in the most deprived quintile of the deprivation index was $40 \%$ higher than in the most affluent quintile. Some malformation subgroups also showed increasing risk with increasing deprivation: all cardiac defects, malformations of the cardiac septa, malformations of the digestive system, and multiple malformations. No evidence for socioeconomic variation was found for other non-chromosomal malformation groups, including neural tube defects and oral clefts. A decreasing risk with increasing deprivation found for all chromosomal malformations and Down's syndrome in unadjusted analyses, occurred mainly as a result of differences in the maternal age distribution between social classes.

Conclusion-Our data, although based on limited numbers of cases and geographical coverage, suggest that more deprived populations have a higher risk of congenital anomalies of non-chromosomal origin and some specific anomalies. Larger studies are needed to confirm these findings and to explore their aetiological implications.

(Arch Dis Child 2000;82:349-352)

Keywords: congenital anomaly; inequalities; social class; deprivation

Congenital anomalies are a major cause of infant mortality and an important contributor to childhood and adult morbidity. The aetiology of the majority of cases of congenital anomaly remains unknown. The presence or absence of socioeconomic inequalities in the prevalence of congenital anomalies, and the extent of such inequalities, can be an important aetiological clue, as it has been for discovering the nutritional aetiology of neural tube defects, ${ }^{1}$ and also determines the potential for socioeconomic confounding in investigations of environmental risk factors such as residence near industrial sites, ${ }^{2}$ or smoking during pregnancy. ${ }^{34}$ The analysis of socioeconomic inequalities in congenital anomaly prevalence is an important component of needs assessment and effective service targeting and is valuable information for the evaluation of population interventions such as the periconceptional folic acid supplementation campaign. $^{5}$

Whereas socioeconomic status has been established as a risk factor for a range of adverse perinatal and infant outcomes such as low birthweight ${ }^{67}$ and perinatal, neonatal, and postneonatal mortality, ${ }^{89}$ surprisingly little literature exists specifically examining socioeconomic inequalities in the prevalence of congenital anomalies.

Studies of all congenital anomalies combined have reported either no clear socioeconomic inequalities ${ }^{10-12}$ or a higher prevalence among lower (status) social classes. ${ }^{13} 14$ Few studies have examined the presence or absence of socioeconomic inequalities in specific congenital defects, with the exception of neural tube defects for which a strong increase in risk among lower social classes has been well documented. ${ }^{1}$ For Down's syndrome both higher $^{13}$ and lower ${ }^{10}{ }^{15}$ prevalences among lower social classes or residents of more socioeconomically deprived areas have been reported, probably mainly secondary to differences in maternal age distribution between the social classes. ${ }^{15} \mathrm{~A}$ number of studies have shown trends of higher risks in lower social classes for oral clefts, ${ }^{136-18}$ which seems to be mainly in relation to cleft palate, ${ }^{13}{ }^{16} 17$ rather than cleft lip. ${ }^{11} 131617$ There are inconsistent reports concerning the existence of social class inequalities for hypospadias. ${ }^{13}{ }^{16}$ Higher prevalences for lower social classes have been reported, by single studies, for congenital cataract, some cardiovascular anomalies, selected genitourinary anomalies, polydactyly, syndactyly, limb reduction defects, and hydrocephalus. ${ }^{13} 161819$ Knox and Lancashire ${ }^{13}$ reported an absence of social class variations for omphalocele/ exomphalos, tracheo-oesophageal fistula, anal atresia, diaphragmatic hernia, and ocular deformities and a higher risk with higher social class for congenital dislocation of the hip. Dolk et $a l^{20}$ reported no socioeconomic variation in the prevalence of anophthalmia.

In this paper we present data on socioeconomic inequalities in the risk of congenital 
anomalies, focusing on risk of specific anomaly subgroups, and show the importance of further research in this area.

\section{Methods}

We used data from a European multicentre case control study on risk of congenital anomaly in the vicinity of hazardous waste landfill sites. A detailed description of the methodology of this study can be found elsewhere. ${ }^{2}$ We collected cases born within defined geographical study areas and registered in three regional UK congenital malformation registers, Glasgow (198 cases, 1991-92), Northern Region (536 cases, 1986-93), and North Thames West (77 cases, 1990-93), ${ }^{2}$ as well as the England and Wales Down Syndrome Register (Essex Region, 17 cases, 198992; Mersey Region, 30 cases, 1989-93). Cases included all malformed live births, stillbirths, and fetal deaths from 20 weeks gestation, and terminations of pregnancy following prenatal diagnosis. Cases with neoplasms, metabolic diseases, familial syndromes, minor malformations, and deformations were not included. Controls were non-malformed live births, approximately two per case, selected from the same year of birth and study area as the case. A total of 858 cases and 1764 controls were studied.

Malformations were classified into nonmutually exclusive subgroups (one case can be included in more than one malformation subgroup), as described previously. ${ }^{2}$ Analyses were carried out for all non-chromosomal anomalies combined, all chromosomal anomalies combined, and malformation subgroups which contained 20 or more cases.

As a measure of socioeconomic status, cases and controls were given a value for the ED level Carstairs deprivation index for Great Britain, ${ }^{21}$ by linking the postcode of residence at birth to census enumeration districts (EDs, areas of approximately 150 households). EDs were grouped into GB quintiles of deprivation, with quintile 1 containing the most affluent areas and quintile 5 the most deprived. Two cases and three controls were born in areas where

Table 1 Risk of congenital anomalies by socioeconomic deprivation-odds ratios for the most deprived compared to the most affluent deprivation quintile

\begin{tabular}{lrrl}
\hline Malformation group & $n$ & $O R^{\star}$ & $95 \%$ CI \\
\hline All non-chromosomal anomalies & 665 & 1.41 & $1.04-1.91$ \\
Neural tube defects & 107 & 1.23 & $0.63-2.37$ \\
Other central nervous system defects & 33 & 1.34 & $0.41-4.40$ \\
All cardiac malformations & 230 & 1.59 & $0.98-2.59$ \\
$\quad$ Malformations of cardiac chambers and connections & 30 & 1.94 & $0.53-7.13$ \\
Malformations of cardiac septa & 135 & 2.82 & $1.43-5.56$ \\
Malformations of cardiac valves & 74 & 1.49 & $0.66-3.36$ \\
Malformations of great arteries and veins & 77 & 1.04 & $0.48-2.23$ \\
Oral clefts & 73 & 0.95 & $0.44-2.05$ \\
Cleft palate & 29 & 0.95 & $0.29-3.09$ \\
Cleft lip/palate & 44 & 0.97 & $0.36-2.63$ \\
Tracheo-oesophageal anomalies & 20 & 1.53 & $0.29-7.95$ \\
Digestive system anomalies & 44 & 3.53 & $1.11-11.18$ \\
Renal and urinary anomalies & 78 & 1.51 & $0.68-3.35$ \\
Limb reduction defects & 27 & 1.22 & $0.34-4.32$ \\
Abdominal wall defects & 20 & 1.57 & $0.35-7.05$ \\
Multiple malformations $\dagger$ & 56 & 2.58 & $0.95-7.01$ \\
All chromosomal anomalies & 191 & 0.73 & $0.44-1.21$ \\
Down's syndrome & 139 & 0.80 & $0.44-1.45$ \\
\hline
\end{tabular}

^Estimated from log linear model and adjusted for maternal age, year of birth, study region, and distance from landfill site.

†Two or more major malformations. deprivation could not be classified. Ten per cent of controls were born in deprivation quintile $1,39 \%$ in quintile 5 .

The association between deprivation and risk of congenital anomaly was investigated by including deprivation quintile as a numerical variable in logistic regression models. Using the slope parameters produced in these regressions, odds ratios were estimated for quintile 5 (most deprived) compared to quintile 1 (most affluent). The likelihood ratio test was used to test for linear trend in the effect of deprivation. Analyses were controlled for region, year of birth, maternal age $(<30,30-34, \geqslant 35)$, and distance of residence from a landfill site $(<3$ $\mathrm{km}, 3-7 \mathrm{~km}$ ), which had previously been shown to be related to congenital anomaly risk. ${ }^{2}$

\section{Results}

We found a greater risk of non-chromosomal anomalies with increasing socioeconomic deprivation. The risk in the most deprived quintile of the deprivation index was $40 \%$ higher than in the most affluent quintile after adjustment for potential confounding factors (adjusted odds ratio (OR) 1.41; 95\% confidence interval (CI) 1.04 to 1.91 ; table 1). Adjustment for confounders did not substantially change the unadjusted OR estimate (unadjusted OR 1.33; 95\% CI 1.01 to 1.75). Most non-chromosomal malformation subgroups, with the exception of oral clefts, showed raised odds ratios in the most deprived compared to the most affluent areas. However, confidence intervals were wide and included unity in most subgroups. Statistically significant trends of increasing risk with increasing deprivation were found only for malformations of the cardiac septa and malformations of the digestive system $(p<0.05)$. All cardiac defects and multiple malformations showed trends of borderline statistical significance $(\mathrm{p}=0.06)$.

Statistically significant trends of decreasing risk with increasing deprivation were found in unadjusted analyses for Down's syndrome (OR quintile 5 versus $1: 0.47,95 \%$ CI 0.29 to 0.76 ) and all chromosomal malformations combined (OR $0.46,95 \%$ CI 0.30 to 0.70 ). Maternal age is a strong potential confounding factor in this relation. The percentage of older mothers (30 years or over) was higher in more affluent areas: $46 \%$ (including $10 \%$ over 35 years) in the most affluent quintile compared to $19 \%$ (including $5 \%$ over 35 years) in the most deprived quintile $(\mathrm{p}<0.0001)$. After adjustment for maternal age the odds ratio for the most deprived versus the most affluent quintile was 0.80 (95\% CI 0.44 to 1.45 ) for Down's syndrome and 0.73 (95\% CI 0.44 to 1.21 ) for all chromosomal malformations combined (table 1). More finely stratified adjustment for maternal age $(<30$, $30-34,35-37,38-40,>40$ ) did not bring the odds ratios closer to unity.

\section{Discussion}

Our data show socioeconomic inequalities in the risk of congenital anomalies of nonchromosomal origin and some specific anomalies with an overall $40 \%$ excess (95\% CI $4 \%$ to $91 \%$ ) in the most deprived areas compared to 
the most affluent areas. We estimate that if the whole population could achieve the health experience of the most affluent quintile, $18 \%$ of congenital anomalies might be avoided. Studies using individual social class based on parental occupation have shown risk ratios of up to 1.6 for the highest compared to the lowest (status) social classes. ${ }^{14}$ We have shown in previous work on low birthweight that area based deprivation (using the Carstairs index) can reveal inequalities as large, if not larger than social class based on individual parental occupation. ${ }^{22}$ Risk ratios for the most deprived compared to the most affluent quintiles of the GB Carstairs deprivation index range from 1.4 for neonatal mortality and 1.6 for stillbirths and low birthweight, to 2.0 for postneonatal mortality and 2.2 for sudden infant deaths. ${ }^{22}{ }^{23}$ Congenital anomalies as a whole thus appear to be among the less socioeconomically determined of the various perinatal and infant outcomes.

We find a more than twofold increase in risk of Down's syndrome and all chromosomal anomalies combined in the most affluent compared to the most deprived areas in analyses unadjusted for confounding factors. Thirty per cent of cases of chromosomal anomaly had mothers resident in more affluent areas (deprivation quintile 1 and 2), compared to $18 \%$ of cases of non-chromosomal origin. After maternal age adjustment, a weak, non-statistically significant, trend of increasing risk with increasing affluence remains for chromosomal anomalies. A previous study in Glasgow also found that controlling for maternal age weakened but did not completely annul a trend of higher risk in more affluent areas. ${ }^{15}$ The finding of a higher risk in more affluent populations, adjusting for maternal age, may result from socioeconomically related environmental exposures differentially affecting intrauterine survival of fetuses with chromosomal anomalies. ${ }^{24}$ This finding could also result if pregnant women in more affluent areas were offered or underwent more prenatal screening for Down's syndrome. This would artificially inflate their apparent risk by counting among terminations cases who would otherwise have resulted in unregistered spontaneous abortions. However, we found no evidence for this when we examined our data for socioeconomic variation in the proportion of chromosomal and Down's syndrome cases that were terminations of pregnancy, adjusting for maternal age.

Numbers of cases for malformation subgroup analyses were small and only tentative conclusions can be drawn from these results. However, the strong socioeconomic gradient found for cardiac septal defects, with a nearly threefold increase in risk in the most deprived compared to the most affluent populations, should be noted. Socioeconomic trends of higher risks in lower social classes have been reported in the literature for congenital heart disease, ${ }^{13}$ ventricular septum defects, ${ }^{16}$ and some specific cardiac defects. ${ }^{19}$ There are strong suggestions that a range of environmental risk factors may be important in the aetiology of cardiac defects, and further research is clearly indicated. In our data digestive system defects also show a strong trend of increasing risk with increasing deprivation although confidence intervals are wide. Knox and Lancashire ${ }^{13}$ report no socioeconomic inequalities for anal atresia which forms $27 \%$ of our group of digestive system defects, and to our knowledge there are no other studies of this group, which also includes anomalies of the small intestine, large intestine, liver, gall bladder, and stomach. The 2.5-fold risk increase of borderline statistical significance we find for multiply malformed infants in more deprived populations is of interest, as most potent teratogens cause multiple rather than isolated malformations..$^{25}$ No other studies have reported on socioeconomic variation in multiple congenital anomalies. Oral clefts, both cleft palate and cleft lip, showed no variation with deprivation in our data, whereas other reports have been fairly consistent in reporting higher prevalences in lower social classes for oral clefts, particularly for cleft palate. ${ }^{13}{ }^{16-18}$

Our data suggest only a very slight gradient of increasing risk with increasing deprivation for neural tube defects, if any, although the confidence intervals again are wide. Social class has however been a well documented risk factor for these defects. Two- to fourfold higher risks in lower social classes have been documented up to the mid-1970s. ${ }^{1}$ There has been little recent work, except a Californian study which found an excess risk of neural tube defects in women from more deprived neighbourhoods in the period 1989-91. ${ }^{26}$ Further research in recent time periods is particularly important in evaluating the impact periconceptional folate supplementation recommendations may have in either increasing or decreasing socioeconomic inequalities in prevalence of neural tube defects. ${ }^{27} 28$

Ascertainment bias within regions, between areas of differing socioeconomic deprivation, seems unlikely to explain our results and the different findings for different anomalies. The registers used multiple sources of information and active case finding and data were collected routinely without knowledge of the study hypotheses. Minor anomalies, for which ascertainment can be especially variable, were not included in the study. Future study should nevertheless ideally investigate the potential for ascertainment bias by hospital of birth.

The aetiology of congenital anomalies is still largely unknown. Our findings, although based on limited numbers of cases and geographical coverage, suggest that risk factors linked to socioeconomic status may play a role in some but not other malformations. Risk factors which could mediate the impact of socioeconomic status on the prevalence of congenital anomalies include nutritional factors, lifestyle, environmental and occupational exposures, access to and use of health services, parity and maternal age, and ethnic origin. In order to close the gap in our knowledge on the extent of socioeconomic differentials in the prevalence of congenital anomalies and how they might be explained, the current findings require follow up in larger studies. 
The work for this paper was carried out under a Research Fellowship for Martine Vrijheid from The Colt Foundation. Coordination of the EUROHAZCON study was funded by the European Commission DGXII BIOMED programme Concerted Action Contract BHM-94-1099. We are grateful to colleagues from the participating registries and to laboratories contributing data to the Down Syndrome Register.

1 Little J, Elwood H. Socio-economic status and occupation. In: Elwood JM, Little J, Elwood H, eds. Epidemiology and control of neural tube defects. Oxford: Oxford University Press, 1992.

2 Dolk H, Vrijheid M, Armstrong B, et al. Risk of congenital anomalies near hazardous-waste landfill sites in Europe: the EUROHAZCON study. Lancet 1998;352:423-7.

3 Khoury MJ, Weinstein A, Panny S, et al. Maternal cigarette smoking and oral clefts: a population-based study. $A m \mathcal{F}$ Public Health 1987;77:623-5.

4 Werler MM. Teratogen update: smoking and reproductive outcomes. Teratology 1997;55:382-8.

5 Health Education Authority. Changing preconceptions volume 1: the HEA Folic Acid Campaign 1995-1998. Summary report. London: HEA, 1998.

6 Reading R, Raybould S, Jarvis S. Deprivation, low birth Reading R, Raybould's, Jarvis S. Deprivation, low birth weight, and children's height: a comparison

7 Leon DA. Influence of birth weight on differences in infant mortality by social class and legitimacy. BMf 1991;303: mortality

8 McIntosh Gray A. Inequalities in health. The Black Report: a summary and comment. Int $\mathcal{F}$ Health Serv 1982;12:34980.

9 Office for National Statistics. Mortality statistics. Childhood, infant and permatal. 1993 and 1994. London: The Stationary Office, 1996

10 Stone DH, Womersley J. Distribution of congenital anomalies within a city: associations with housing type. Eur 7 Epidemiol 1989;5:255.

11 Ericson A, Eriksson M, Zetterstrom R. The incidence of congenital malformations in various socioeconomic groups in Sweden. Acta Paediatr Scand 1984;73:664-6.

12 Tuohy PG, Counsell AM, Geddis DC. The Plunket National Child Health Study: birth defects and socioNational Child Health Study: birth defects and

13 Knox EG, Lancashire RJ. Frequencies and social variations. Epidemiology of congenital malformations. London: HMSO, 1991.
14 Olsen J, Frische G. Social differences in reproductive health. Scand F Soc Med 1993;21:90-7.

15 Lopez PM, Stone D, Gilmour H. Epidemiology of Down's syndrome in a Scottish city. Paediatr Perinat Epidemiol 1995;9:331-40

16 Olshan F, Baird PA, Lo KH. Socioeconomic status and the risk of birth defects. Am ₹ Epidemiol 1991;134:778-9.

17 Womersley J, Stone DH. Epidemiology of facial clefts. Arch Dis Child 1987;62:717-20.

18 Hemminki K, Mutanen P, Luoma K, Saloniemi I. Congenital malformations by the parental occupation in Finland. Int Arch Occup Environ Health 1980;46:93-8.

19 Correa-Villasenor A, McCarter R, Downing J, Ferencz C, Group B-WIS. White-black differences in cardiovascular malformations in infancy and socioeconomic factors. Am $\mathcal{F}$ Epidemiol 1991;134:393-402.

20 Dolk H, Busby A, Armstrong BG, Walls PH. Geographical variation in anophthalmia/microphthalmia in England, 1988-1994. BMF 1998;317:905-10.

21 Carstairs V, Morris R. Deprivation and health in Scotland. Health Bull (Edinb) 1991;48:162-75.

22 Pattenden S, Dolk H, Vrijheid M. Inequalities in low birthweight: parental social class, area deprivation and "lone mother" status. F Epidemiol Community Health 1999; 53:355-8.

23 Dolk H, Pattenden S, Vrijheid M, Thakrar B, Armstrong B. Perinatal and infant mortality and low birthweight among residents near cokeworks in Great Britain. Arch Environ Health 2000 [In press]

24 Hook EB, Cross PK. Maternal cigarette smoking, Down syndrome in live births, and infant race. Am f Hum Genet 1988;42:482-9.

25 Dolk H, de Wals P. Congenital anomalies. In: Elliott P, Cuzick J, English D, Stern R, eds. Geographical and environmental epidemiology: methods for small area studies. Oxford: tal epidemiology: methods for small area
Oxford University Press, 1992:72-88.

26 Wasserman CR, Shaw GM, Selvin S, Gould JB, Syme SL. Socioeconomic status, neighborhood social conditions, and neural tube defects. Am f Public Health 1998;88:1674-80.

27 Achenson D. Independent inquiry into inequalities in health. London: The Stationary Office, 1998.

28 De Walle HEK, van der Pal KM, de Jong-van den Berg LTW, et al. Periconceptional folic acid in the Netherlands in 1995. Socioeconomic differences. F Epidemiol Community Health 1998;52:826-7. 